\title{
Assessment of central visual function after successful retinal detachment surgery by pattern visual evoked cortical potentials
}

\author{
Masahiro Ueda, Emiko Adachi-Usami
}

\begin{abstract}
The pattern of visual recovery after successful surgery by pattern visual evoked cortical potentials (VECP), visual acuity, colour vision, and critical fusion frequency was investigated in 14 eyes with retinal detachment involving the macula. The temporal tuning characteristics in the evoked potentials were measured as based on the $\boldsymbol{P}_{100}$ amplitude and the frequency necessary for evoking $0 \mu \mathrm{V}$ amplitude, which was defined as an objective critical fusion frequency by extrapolating the curve. Significant improvement in visual acuity and colour vision was observed within 2 months postoperatively. A significantly increased $\boldsymbol{P}_{100}$ peak latency became shorter as the postoperative days increased. In general, a good correlation was noted between the $\boldsymbol{P}_{100}$ peak latency and subjectively measured visual acuity, colour vision, and critical fusion frequency. The objective critical fusion frequency measured by VECP recovered gradually during the 6 months after surgery. Functional recovery was not related to the length of time the macula was detached before surgery.
\end{abstract}

(Br F Ophthalmol 1992; 76: 482-485)

A discrepancy exists between anatomical recovery after retinal detachment surgery and subjective visual functions as represented by visual acuity and colour vision. Several reports ${ }^{1-4}$ on subjective visual functions after successful retinal detachment surgery have indicated that, although visual function improved gradually within several months after operation, it never returned to the preoperative level. The influencing factor is believed to be duration of macular detachment. ${ }^{412}$

Two methods of measuring central visual function objectively have recently been developed. One is focal macular electroretinography and the other is visual evoked cortical potentials (VECP) to pattern stimulation. The latter reflects central visual function, ${ }^{13}$ without using the complicated retinal stimulus conditions necessary for a local electroretinogram. Surprisingly little is known from the electrophysiological standpoint, particularly using pattern VECPs after retinal detachment surgery.

We evaluated central visual function objectively with the use of pattern VECPs and subjectively with visual acuity, colour vision, and critical fusion frequency in patients with rhegmatogenous retinal detachment involving the macula who underwent successful retinal repair.

\section{Subjects and methods}

Fourteen patients with unilateral rhegmatogenous retinal detachment involving the macula, who had successful anatomical surgical results showing no macular pucker or cystoid macular oedema between December 1989 and July 1990 at our clinic, have been followed-up for 6 months. All subjects who participated in this study were fully informed of the procedures and gave their consent for inclusion. The nine male and five female patients ranged in age from 14 to 65 years (mean age 38.5 years). Of the 14 patients, 13 eyes underwent conventional surgical techniques of scleral buckling with cryopexy and drainage of fluid; one eye was treated with pneumatic retinopexy and light coagulation. The duration of preoperative macular detachment, which was estimated as the time from onset of subjective visual loss to the day of surgery, ranged from 10 to 16 days for nine eyes. Excluding the case representing the longest duration (6 months), the mean duration was $17 \cdot 8$ days. In all cases preoperative visual acuity was 0.2 or less, ranging from 0.01 to 0.2 (mean 0.09 ).

Visual acuity, colour vision, critical fusion frequency, and pattern VECPs were examined at 2 weeks and $1,2,4$, and 6 months after surgery. The fellow eyes were tested as controls. The data were statistically analysed, based on student's $t$ test.

Visual acuity was corrected for refraction with use of the autorefractometer (RK-1, Canon Ltd, Japan) and skiaskopy after administration of atropine. With the appropriate near correction, colour tests were administered monocularly under a level of 800 lux at the test plane given by a 20 W Toshiba EDL-65 fluorescent tube.

The standard pseudochromatic plates, part II, and panel D-15 were used for tests of colour vision. During the follow-up period, all patients passed a panel D-15 test. The results of the pseudochromatic test showed high error scores in misreading the $10 \mathrm{~B}-\mathrm{Y}$ plates. After evaluating the number of $B-Y$ plates misread, we excluded from the test, the 3rd left plate ' 2 ', because this plate was often misread by those with normal colour vision. ${ }^{14}$

Subjective critical fusion frequency was measured with test apparatus II of Osaka University (Yagami Ltd, Japan). The frequency required to perceive a flickering light was determined three times to obtain the mean.

For VECP recordings, the electrode was placed at $\mathrm{O}_{z}$ and referred to the earlobe. The stimulus was a chequerboard pattern displayed on a black and white TV monitor. The pattern reversed at rates of $2,4,6,8,10,12,15,20$, and 


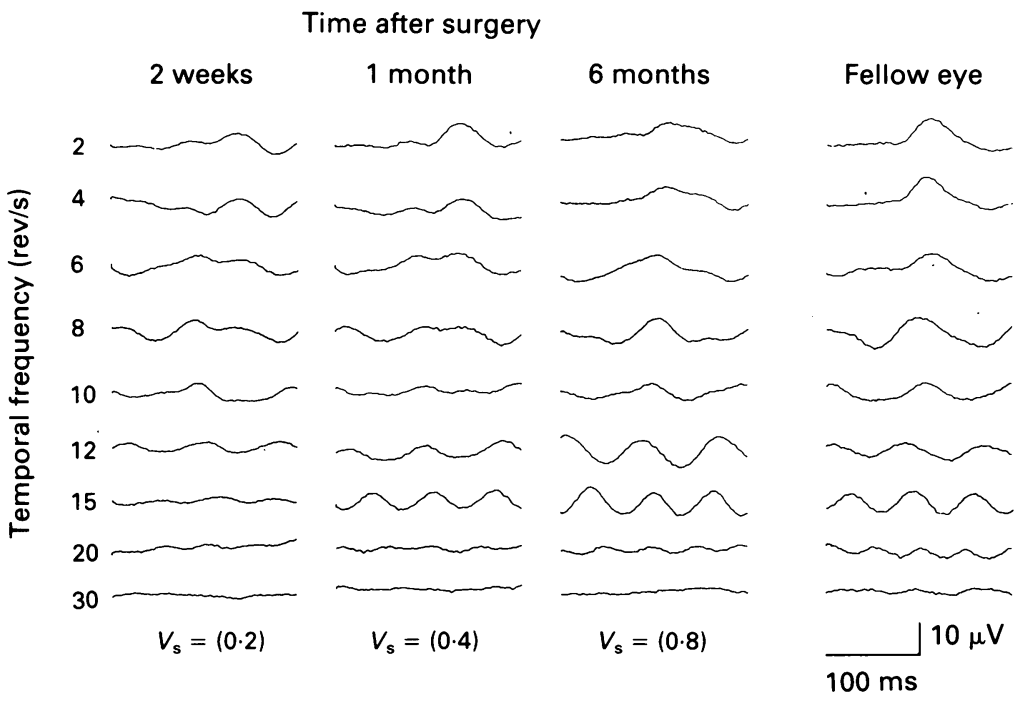

Figure 1 Actual pattern visual evoked cortical potential recordings after surgery in a 54-year-old female patient with rhegmatogenous retinal detachment involving the macula. ing distance of $180 \mathrm{~cm}$ Contrast and mean luminance were kept at $80 \%$ at 0.59 (SE 0.09) at 6 months. This level was significantly lower than that of the non-affected fellow eyes $(1 \cdot 10($ SE 0.07)) $(p<0.01)$.

Postoperative maximal visual acuity had no correlation with the duration of preoperative macular detachment (Fig 4).

\section{COLOUR VISION}

All of the non-affected fellow eyes of the patients passed both tests of colour vision. All of the affected eyes examined at 2 weeks after surgery passed the panel D-15 test. Nine of 14 eyes misread the B-Y plates of the pseudochromatic test at 2 weeks postoperatively, and underwent a repeated testing.

The mean number of those misreading the B-Y plates at five different times after surgery is shown in Figure 5. The number decreased significantly $(p<0.05)$ from 2 weeks to 2 months after surgery. Four eyes were still misreading the B-Y plates at 6 months after surgery. and $41.5 \mathrm{~cd} / \mathrm{m}^{2}$, respectively. The patient fixed on a point in the centre of the pattern monocularly. The pupil was dilated, and an artificial pupil of $3 \mathrm{~mm}$ was used. One hundred responses were averaged (ATAC 350, Nihon Kohden Ltd, Tokyo), after passing the pre-amplifier (AVB9, Nihon Kohden Ltd, Tokyo) with a bandpass of 1.5 to $100 \mathrm{~Hz}$, and printed by an X-Y recorder, in the positivity of the scalp electrode with an upward deflection (Fig 1). Peak latency of the first positive component $\left(P_{100}\right)$ was measured in response to the transient stimulus of $2 \mathrm{rev} / \mathrm{s}$.

The amplitude of the cortical potentials was measured between the top of the $P_{100}$ component and the bottom of the following negative wave for the transient stimulus. For steady state potentials, the average of the peak-to-trough amplitude of sinusoidal waves was determined. Amplitudes were plotted as a function of temporal frequency on a $\log$ scale. The inverse U-shaped curve was obtained for each frequency range examined. At higher frequency ranges the amplitude decreased; a linear relationship existed between the amplitude and increasing log stimulus reversal frequency. The regression line was calculated, and VECP-critical fusion frequency (CFF) was determined by extrapolating the line to $0 \mu \mathrm{V}$ amplitude ${ }^{15}$ (Fig 2).

\section{Results}

Only visual acuity was,measured preoperatively. Because the retinal detachment involved the macula the eyes were judged to be unable to provide reliable results in the other three test conditions because of poor visual acuity.

\section{VISUAL ACUITY}

The corrected visual acuity before and after operation is shown in Figure 3. It improved significantly up to 1 month after surgery, from a preoperative vision of mean 0.09 (SE 0.02 ) to 0.36 (SE 0.06). Thereafter a slight gradual improvement was observed for up to 2 months after surgery. No further recovery was observed thereafter, however, and visual acuity remained
SUBJECTIVE CRITICAL FUSION FREQUENCY (CFF) Figure 6 showed the mean of CFF after surgery. At 2 weeks postoperatively the frequency was 33.6 (SE 0.9) Hz, which was significantly lower $(\mathrm{p}<0.01)$ than that of the fellow eyes $(41.7$ (SE $0.6 \mathrm{~Hz})$ ). The frequency increased significantly at 1 month to $38.4(\mathrm{SE} 1.2) \mathrm{Hz}(\mathrm{p}<0.01)$, but no further improvement was observed until 6 months postoperatively.

\section{$P_{100}$ PEAK LATENCY}

The mean peak latency of the $P_{100}$ component of the evoked potentials (Fig 7) that was obtained at 2 weeks after surgery was $137 \cdot 5$ (SE $3 \cdot 1$ ) ms was significantly prolonged $(\mathrm{p}<0.01)$, when compared with that of the non-affected fellow eye (116.5 (SE 1.9) ms). The peak latency became shorter with time, but still was prolonged $(p<0.01)$ at 6 months after surgery.

\section{VISUAL EVOKEDCORTICAL POTENTIAL-CRITICAL} FUSION FREQUENCY

We were able to test with certainty the VECPCFF in eight of 14 eyes (Fig 8). Compared with

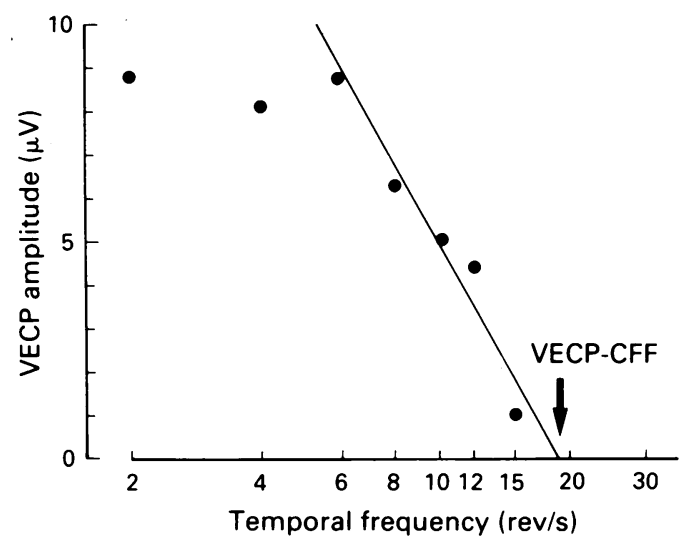

Figure 2 Visual evoked cortical potential (VECP) amplitudes as a function of log stimulus reversal frequency in a 59-year-old female patient. VECP-critical fusion frequency $(V E C P-C F F)$ was determined by extrapolating $a$ regression line at a higher frequency range to $0 \mu \mathrm{V}$. 
Figure 3 Mean (SE) visual acuity before and after surgery. The bars indicate \pm standard errors $(S E)$.

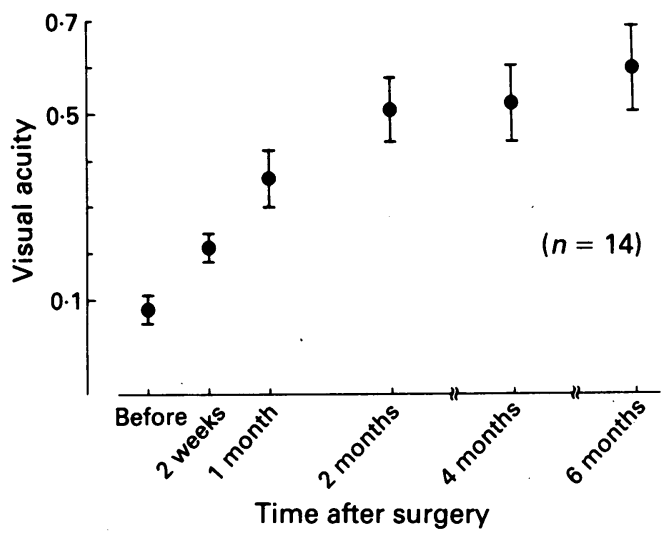

that of the fellow eye $(28 \cdot 1$ (SE 1.7) rev/s), the mean value in the affected eye was significantly lower at 2 weeks $(18 \cdot 1 \mathrm{rev} / \mathrm{s})(\mathrm{p}<0.01)$. Thereafter, it began to increase gradually to 20.3 at 1 month, 22.7 at 2 months, 24.6 at 4 months, and $27.5 \mathrm{rev} / \mathrm{s}$ at 6 months. The increase from 2 weeks to that at 4 months was significant $(\mathrm{p}<0 \cdot 01)$. No significant difference was observed in CFF after 2 months.

\section{Discussion}

After surgical repair ophthalmoscopically reattached retinas do not produce a satisfactory recovery of visual acuity, according to patients. One of the factors thought to influence visual recovery has been duration of preoperative macular detachment, ${ }^{1-12}$ as reported precisely by Burton. ${ }^{12}$

The present study showed no such relation, although the number of cases is relatively low, because of the limited number of patients who could cooperate with electrophysiological measurements. Even the eye with a preoperative macular detachment of 6 weeks' duration improved to a visual acuity of $1 \cdot 2$. Only one eye, with a long-standing macular detachment of 6 months, demonstrated the worst visual acuity of $0 \cdot 2$.

Gundry and Davies ${ }^{1}$ reported on one patient with a 1 day preoperative detachment. The visual acuity in that eye improved to $6 / 9$, which was still lower than that of normal. The average maximal visual acuity after successful surgery showed improvements equal to half that of the level before detachment, which corresponded well with our results. Conversely, Chisholm and associates ${ }^{4}$ reported that a visual acuity of $0 \cdot 1$ was the norm for the first postoperative year and $0 \cdot 17$ by the end of the second year.

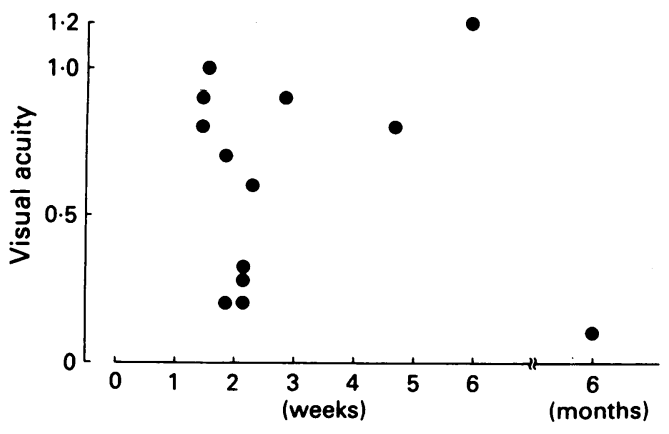

Duration of preoperative macular detachment

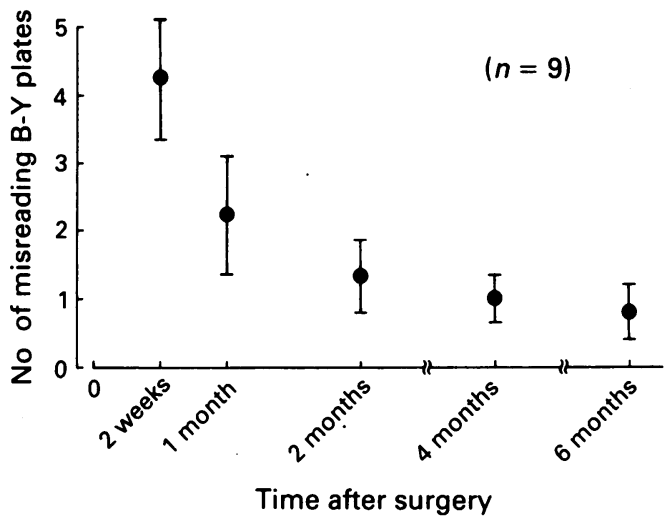

Figure 5 Mean number (SE) of misreading B-Y plates of the standard pseudochromatic test part II after surgery.

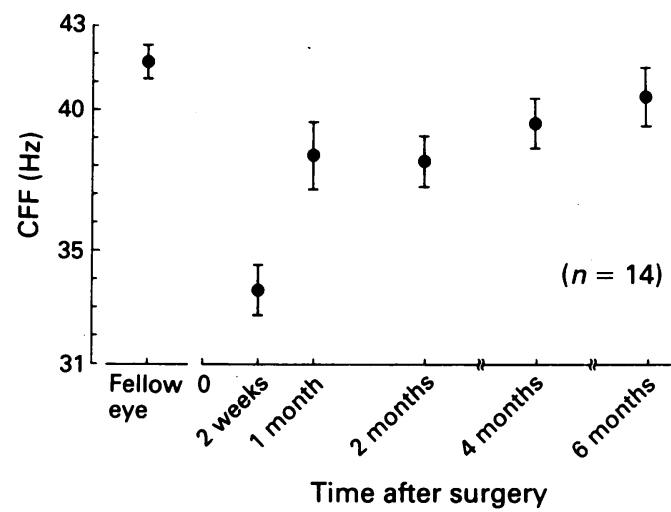

Figure 6 Mean $(S E)$ subjective critical fusion frequency $(C F F)$ after surgery.

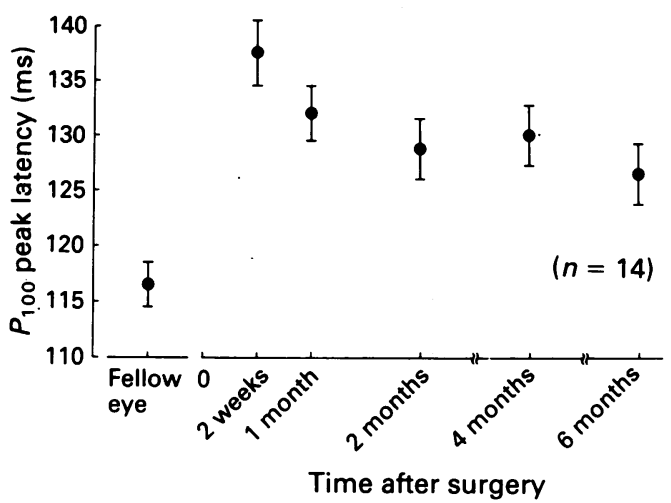

Figure 7 Mean (SE) peak latency of the $\mathbf{P}_{100}$ of pattern visual evoked cortical potentials after surgery.

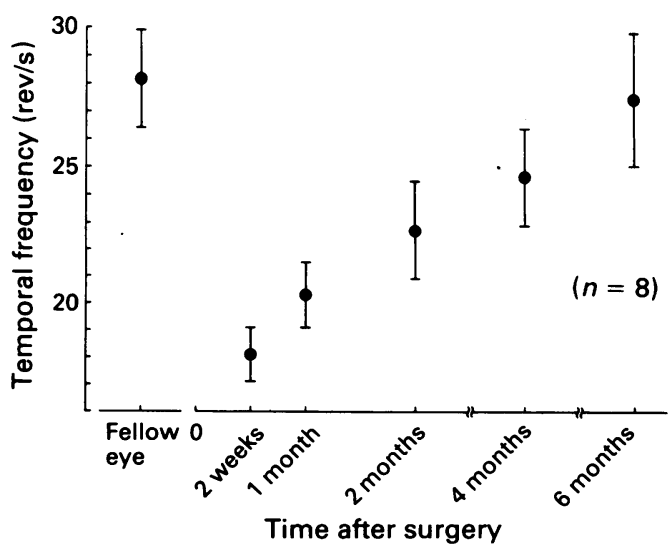

Figure 8 Mean (SE) pattern visual evoked cortical potential-critical fusion frequency $(V E C P-C F F)$ after surgery. 
The time required for a steady maximum visual acuity was found to be within 2 months, as reported by Gundry and Davies, ${ }^{1}$ Foulds $e t a l,{ }^{2}$ Binder et $a l,{ }^{3}$ and Burton. ${ }^{12}$

Colour vision after surgery has shown B-Y defects, as based on the FM 100-hue test. ${ }^{1-3} \mathrm{We}$ also found B-Y defects with the use of the pseudochromatic test, which was sensitive for evaluating acquired colour vision deficiency. ${ }^{14}{ }^{16}$ Recovery of the B-Y defect was similar to that of visual acuity; B-Y defects recovered within 2 months to the preoperative level, with a residual defect. Foulds $e t a^{2}$ described a residual defect of hue discrimination that lasted for 2 years in those eyes which were followed up.

To our knowledge no report has tested both subjective and objective critical fusion frequency in follow-up studies of retinal detachment. In the present study, we showed less decrease and faster recovery of subjective critical fusion frequency in comparison with visual acuity and colour vision, although the frequency reflects visual function from the ganglion cell layer of the retina to the visual cortex.

To test central visual functions objectively, either focal macular electroretinography or visual evoked cortical potentials can be used at present. A focal electroretinogram requires a fine recording set-up to avoid the effects of stray light. On the other hand, pattern visual evoked cortical potentials are gradually becoming popular in clinical use to examine central vision from the macular region to the visual cortex.

Using pattern evoked cortical potentials, we found that the prolongation of the $P_{100}$ peak latency after surgery shortened with the improvement of visual acuity and colour vision, and that the evoked potentials-critical fusion frequency based on the amplitude increased with recovery of visual acuity. We have to be aware of the importance of pattern evoked cortical potentials as a central visual function test.

With regard to the pathological features of the reattached retina, several series of experiments have been described by Machemer's group. ${ }^{17-19}$

Machemer, ${ }^{17}$ and Kroll and Machemer ${ }^{18}$ experimentally induced retinal detachment in the owl monkey and observed the anatomical findings in the reattached retina after surgery. They demonstrated that the retina became normal within 8 weeks after regeneration and reposition of receptor cell outer segments. In another study, ${ }^{19}$ the authors used the rhesus monkey to show the rod repositioned within 1 month, which was an earlier response compared with the cone. Our results, based on both subjective and objective measurements, suggested lesser functional recovery of receptor cells at the central retina.

Sarin and McDonald ${ }^{20}$ found optic atrophy secondary to vascular occlusion after reattachment procedures. As far as our pattern evoked potential results are concerned minor pathologi- cal changes of the optic nerve were suggested although none was observed ophthalmoscopically.

Reduced functional recovery after successful surgery might be due not only to the anatomical changes but also to biochemical disorders, such as decreased neurotransmitters in the visual pathway. Delay of pattern evoked potentials has been found in parkinsonian patients who lack dopamine $^{21}$ and in elderly subjects who have reduced levels of dopamine. ${ }^{22}$ The species difference between the monkey and human might explain the discrepant findings.

This study was presented at the 29th meeting of the International Society of Clinical Electrophysiology of Vision, Oxford, England, 5 July 1991

We thank Ms Maxine Gere for editing the manuscript.

1 Gundry MF, Davies EN, Recovery of visual acuity after retinal detachment surgery. Am $\mathcal{F}$ Ophthalmol $1974 ; 77: 310-4$

2 Foulds WS, Reid H, Chisholm IA. Factors influencing visual recovery after retinal detachment surgery. Mod Probl Ophthalmol 1974; 12: 49-57.

3 Binder S, Kutschera E, Weiss H. Die Makulafunktion nach postoperativer Wiederanlegung der Netzhaut. Klin Monatsbl Augenheilkd 1977; 171: 606-10.

4 Chisholm IA, McClure E, Foulds WS. Functional recovery of the retina after retinal detachment. Trans Ophthalmol Soc UK 1975; 95: 167-72.

5 Norton EWD. Retinal detachment in aphakia. Trans Am Ophthalmol Soc 1963; 61: 770-89.

6 Grupposo SS. Visual results after scleral buckling with silicone implant. In: Schepens CL, Regan CDJ, eds. Controversial aspects of the management of retinal detachment. Boston: Little aspects of the management
Brown, 1965: 354-63.

7 Hilton GF, Norton EWD, Curtin VT, et al. Retinal detachment surgery: a comparison of diathermy and cryosurgery. Mod Probl Ophthalmol 1969; 8: 440-8.

8 Davies EWG. Factors affecting recovery of visual acuity following detachment of the retina. Trans Ophthalmol Soc UK 1972; 92: 335-44.

9 Kaufman PL. Prognosis of primary rhegmatogenous retinal detachments. Acta Ophthalmol (Kbh) 1976; 54: 61-74.

10 Kreissig I: Prognosis of return of macular function after retinal reattachment. Mod Probl Ophthalmol 1977; 18: 415-29.

11 Tani P, Robertson DM, Langworthy A. Prognosis for central vision and anatomic reattachment in rhegmatogenous retinal 92: 611-20.

12 Burton TC. Recovery of visual acuity after retinal detachment involving the macula. Trans Am Opthalmol Soc 1982; 80: involving

13 Spekreijse H. Analysis of EEG responses in man evoked by sine wave modulated light. The Hague: Dr W Junk, 1966.

14 Tsukamoto $M$; Adachi-Usami E. Comparative studies among standard pseudochromatic plates. Part 2. Lanthony's new color test and panel D-15 tests in various acquired color vision deficiencies. Acta Soc Ophthalmol fpn 1986; 90: 65764.

15 Adachi-Usami E. Human visual system modulation transfer function measured by evoked potentials. Neurosci Lett 1981 23: 43-7.

16 Tsukamoto M, Adachi-Usami E, Fujimoto N. Color vision estimated by color pattern reversal VECPs in patients with intraocular lens implant. In: Ohta Y, ed. Color vision

17 deficiencies. Amsterdam: Kugler \& Ghedini, 1990: 77-85. Machemer R. Experimental retinal detachment in the owl
monkey. IV. The reattached retina. Am $\mathcal{F}$ Ophthalmol 1968; monkey. IV. The

18 Kroll AJ, Machemer R. Experimental retinal detachment in the owl monkey. V. Electron microscopy of the reattached retina. Am f Ophthalmol 1969; 67: 117-30.

19 Kroll AJ, Machemer R. Experimental retinal detachment and reattachment in the rhesus monkey. Electron microscopic comparison of rods and cones. Am $\mathcal{F}$ Ophthalmol 1969; 68: 58-77.

20 Sarin LK, McDonald PR. Changes in the posterior pole following successful reattachment of the retina. Trans Am Acad Ophthalmol Oto-Laryngol 1970; 74: 75-9.

21 Bodis-Wollner I, Yahr MD. Measurement of visual evoked potentials in Parkinson's disease. Brain 1978; 101: 661-71.

22 Adachi-Usami E. Senescence of visual function as studied by visually evoked cortical potentials. $\mathcal{F}$ pn $\mathcal{F}$ Ophthalmol 1990 ; 34: 81-94. 